\title{
Oral and cloacal microflora of wild crocodiles Crocodylus acutus and $C$. moreletii in the Mexican Caribbean
}

\author{
Pierre Charruau ${ }^{1, *}$, Jonathan Pérez-Flores ${ }^{2}$, José G. Pérez-Juárez ${ }^{2}$, \\ J. Rogelio Cedeño-Vázquez ${ }^{3}$, Rebeca Rosas-Carmona ${ }^{3}$ \\ ${ }^{1}$ Departamento de Zoología, Instituto de Biología, Universidad Nacional Autónoma de México, Distrito Federal 04510, Mexico \\ ${ }^{2}$ Departamento de Salud y Bienestar Animal, Africam Safari Zoo, Puebla, Puebla 72960, Mexico \\ ${ }^{3}$ Departamento de Ingeniería Química y Bioquímica, Instituto Tecnológico de Chetumal, Chetumal, Quintana Roo 77013, Mexico
}

\begin{abstract}
Bacterial cultures and chemical analyses were performed from cloacal and oral swabs taken from 43 American crocodiles Crocodylus acutus and 28 Morelet's crocodiles C. moreletii captured in Quintana Roo State, Mexico. We recovered 47 bacterial species $(28$ genera and 14 families) from all samples with $51.1 \%$ of these belonging to the family Enterobacteriaceae. Fourteen species $(29.8 \%)$ were detected in both crocodile species and $18(38.3 \%)$ and $15(31.9 \%)$ species were only detected in American and Morelet's crocodiles, respectively. We recovered 35 bacterial species from all oral samples, of which $9(25.8 \%)$ were detected in both crocodile species. From all cloacal samples, we recovered 21 bacterial species, of which $8(38.1 \%)$ were detected in both crocodile species. The most commonly isolated bacteria in cloacal samples were Aeromonas hydrophila and Escherichia coli, whereas in oral samples the most common bacteria were $A$. hydrophila and Arcanobacterium pyogenes. The bacteria isolated represent a potential threat to crocodile health during conditions of stress and a threat to human health through crocodile bites, crocodile meat consumption or carrying out activities in crocodile habitat. We especially warn about the presence of Salmonella arizonae and S. typhi, which cause enteritis and septicemia in crocodiles and salmonellosis and typhoid fever in humans. The risk of bacterial contamination from crocodiles to humans could increase in the future because of the accelerated destruction of crocodile habitat, which could lead to an augmentation of human-crocodile interactions. Information on bacterial diversity reported here could help in the choice of antibacterial products in case of infections that are of crocodile origin.
\end{abstract}

KEY WORDS: Bacterial flora $\cdot$ Crocodylus acutus $\cdot$ Crocodylus moreletii $\cdot$ Salmonella $\cdot$ Cozumel Río Hondo $\cdot$ Banco Chinchorro $\cdot$ Mexico

\section{INTRODUCTION}

Crocodilians have the strongest bite of all living animals (Erickson et al. 2003) and can cause serious injuries. The gravity of wounds ranges from simple punctures or tearing skin wounds to fractures, amputations and death (Caldicott et al. 2005, Hertner 2006, Gruen 2009, Cupul-Magaña et al. 2010, Langley 2010). Conflicts between humans and crocodilians have been reported in many countries and while in some regions attacks on humans are uncommon, in others they seem to occur frequently (Pooley et al. 1989, Lamarque et al. 2009, Wamisho et al. 2009). Those adverse encounters are expected to increase in the future because of the modification and destruction of crocodile habitat or the increase in crocodile numbers (Scott \& Scott 1994, Caldicott et al. 2005, Vyas 2010). 
Besides the physical damage, survivors of attacks from crocodilians can develop wound infections caused by bacteria or fungi (Flandry et al. 1989, Caldicott et al. 2005, Wamisho et al. 2009, Langley 2010). Several studies display the existence of many bacteria and fungi in the oral cavity of wild and captive crocodilians (Doering et al. 1971, Gorden et al. 1979, Flandry et al. 1989, Matushima \& Ramos 1995, Anderson 1999, Cupul-Magaña et al. 2005, Silva et al. 2009). While several antibiotics provide protection from wound infections, in some cases patients can die from sepsis (Vanwersch 1998, Caldicott et al. 2005). Crocodilians show great resistance and healing capacity to injuries and illness, but several studies report diseases or symptoms in crocodilians related to bacterial infections, which can even lead to the death of individuals (Shotts et al. 1972, Novak \& Seigel 1986, Hernández-Hurtado \& Cupul-Magaña 1996, Mehrotra et al. 2000, Huchzermeyer 2003, Bishop et al. 2007, Garcia et al. 2008).

The Quintana Roo State is the major tourist region in Mexico, where increasing coastal development continues to destroy and fragment the habitats of the American crocodile Crocodylus acutus and Morelet's crocodile C. moreletii (Cedeño-Vázquez et al. 2006, Machkour-M'Rabet et al. 2009). This reduction of habitat, along with other factors, is causing the depletion of crocodile populations and could also increase human-crocodile encounters (Cupul-Magaña et al. 2010). Several cases of crocodile attacks have been reported in Quintana Roo and are often linked to fishing and spear-fishing activities (Lazcano-Barrero 1996, Cantera 2000).

Although the number of studies on crocodiles has increased in the last decade in Quintana Roo, they have yet to examine the bacterial flora of crocodiles in this region. The objective of the present study was to determine the bacterial flora in oral and cloacal cavities of wild American and Morelet's crocodiles in Quintana Roo. This information would then serve in the assessment of health risks to humans after encounters with crocodiles.

\section{MATERIALS AND METHODS}

\section{Study site}

Samples were obtained from American crocodiles captured on Cozumel Island and Banco Chinchorro Biosphere Reserve, and from Morelet's crocodiles captured in the Río Hondo. In Cozumel, crocodiles were captured at Colombia lagoon, Chunchaka'ab lagoon and Xtakún lagoon in the Ecological Reserve of Punta Sur in the southern tip of the Island (Fig. 1). Banco Chinchorro is an atoll located $30 \mathrm{~km}$ east from the coast of Quintana Roo and crocodiles were captured at Cayo Centro, the largest Cay of the atoll (Fig. 1). These reserves have relatively well-conserved populations of American crocodiles with individuals of all size classes and with male-biased sex ratios (Charruau et al. 2005, 2010, González-Cortés 2007). The Río Hondo is a relatively deep river (mean depth of about $8 \mathrm{~m}$ ) that forms the border between México and Belize in South Quintana Roo (Fig. 1). Cedeño-Vázquez et al. (2006) found a relatively high number of Morelet's crocodiles in the Río Hondo with individuals of all size-classes and equal sex ratio and encounter rates that ranged from 1.72 to 4.70 crocodiles $\mathrm{km}^{-1}$.

\section{Sample collection}

Crocodiles were captured at night by hand or by using the break-away snare technique, depending on their size, during spotlight surveys in May 2008 and May 2009 in Cozumel, in August 2010 in Banco Chinchorro and from June 2009 to February 2010 in Río Hondo. Each captured individual was physically examined for signs of diseases, sex was determined by cloacal examination (Brazaitis 1968, Ziegler \& Olbort 2007) and each was measured from the tip of the snout to the tip of the tail $( \pm 0.5 \mathrm{~cm})$. Crocodiles were classified by size class based on their total length (TL). We used the following classes for American crocodiles: hatchlings (TL $<30 \mathrm{~cm}$ ), yearlings $(\mathrm{TL}=30.1$ to $60 \mathrm{~cm})$, juveniles $(\mathrm{TL}=60.1$ to $120 \mathrm{~cm})$, subadults $(\mathrm{TL}=120.1$ to $180 \mathrm{~cm}$ ) and adults $(\mathrm{TL}>$ $180 \mathrm{~cm})$. For Morelet's crocodiles, we used the following classes: hatchlings ( $\mathrm{TL}<30 \mathrm{~cm}$ ), yearlings (TL $=30.1$ to $50 \mathrm{~cm})$, juveniles $(\mathrm{TL}=50.1$ to $100 \mathrm{~cm})$, subadults $(\mathrm{TL}=100.1$ to $150 \mathrm{~cm}$ ) and adults $(\mathrm{TL}>$ $150 \mathrm{~cm}$ ). Samples were taken by passing a sterile culture swab $\left(\mathrm{BBL}^{\mathrm{TM}}\right.$ CultureSwab ${ }^{\mathrm{TM}}$ Plus, Becton-Dickinson) in the oral cavity and the cloaca of each crocodile. Swabs were then preserved at 2 to $8^{\circ} \mathrm{C}$ in AMIES transport mediums (Becton, Dickinson), which are ideal for aerobic and anaerobic bacteria, and were sent for analyses to the Africam Safari Zoo laboratory in Puebla, Mexico.

\section{Bacterial analyses}

Bacterial cultures were grown on various bacterialgrowth media (Cowan \& Steel 1974, Quinn et al. 


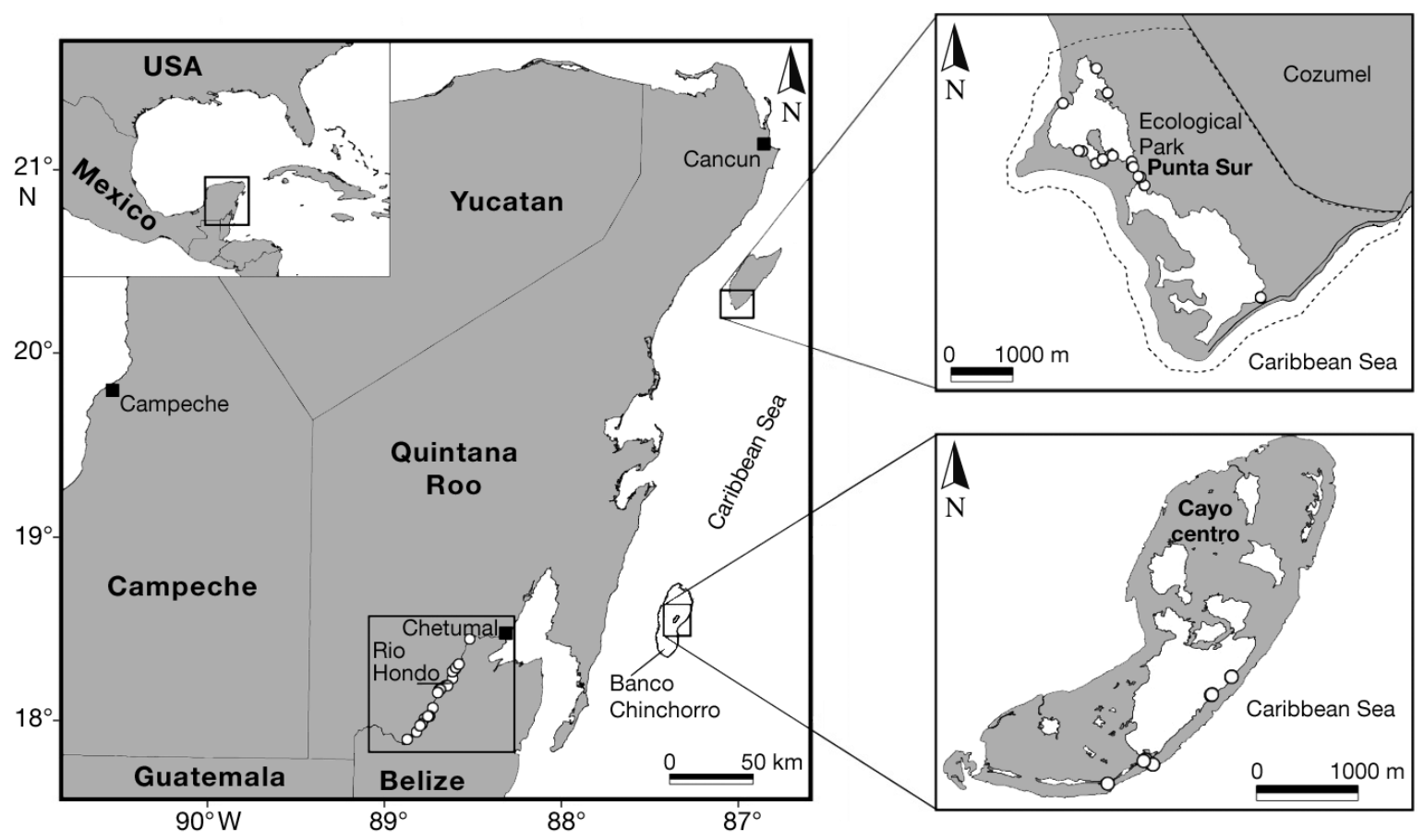

Fig. 1. Crocodylus acutus and C. moreletii. Location of study areas and capture sites (open circles) of American and Morelet's crocodiles in Quintana Roo State. Inset in left panel shows location of study areas in Mexico. Dotted line in upper right panel indicates boundary of ecological park

1994): blood agar $5 \%$, McConkey agar, mannitol salt agar, brilliant green agar, and Salmonella-Shigella agar.

The quadrant streak method was used to inoculate whole plates. These plates were then incubated at $37^{\circ} \mathrm{C}$ for $24 \mathrm{~h}$. Plates of blood agar were incubated in a carbon dioxide atmosphere of $5 \%$ and the other plates were incubated in aerobic conditions (Quinn et al. 1994). After $24 \mathrm{~h}$ of incubation, bacteria morphology was analyzed microscopically and by gross observation to record characteristics such as color, size, form, hemolysis, pigment production, smell and reaction to the indicators of the differential medium. Subsequently, each bacterial colony was submitted to catalase and oxidase tests and a smear from each colony was heat-fixed for Gram staining. If it was necessary to purify the strains, a second inoculation was performed and for the next $24 \mathrm{~h}$ these plates were subjected to the same procedures as were the first inoculations.

After gross observations, we proceeded to identify the Gram-negative bacteria by means of the following biochemical tests: mobility, presence of indole, decarboxylation of ornithine and lysine, fermentation of lactose, sucrose and dextrose, oxidation of iron, use of citrate as a source of carbon, hydrolysis of urea, gas production, deamination of phenylalanine, nitrate reduction and differentiation of oxidative or reductive metabolism of bacteria (Kämpfer et al. 1991, MacFaddin 2000). All these reactions, along with the characteristics obtained during the primary identification, were compared with reference tables of biochemical reactions to obtain the final identification (Cowan \& Steel 1974, Quinn et al. 1994, Murray et al. 1999).

For Gram-positive and Gram-negative bacteria requiring additional tests for identification we used a miniaturized method with the help of the BBL Crys$\mathrm{tal}^{\mathrm{TM}}$ Enteric/Non-fermenter Identification System and the BBL Crystal ${ }^{\mathrm{TM}}$ Rapid Gram-positive Identification System (Becton-Dickinson), both of which contain 30 dried biochemical and enzymatic substrates. Fermentation reactions detect the ability of an isolate to metabolize carbohydrates in the absence of atmospheric oxygen, and oxidation reactions are based on the ability of an organism to metabolize the substrate with oxygen as the final electron acceptor. Both reactions are usually detected by means of a $\mathrm{pH}$ indicator in the test substrate (Killian \& Bulow 1976, MacFaddin 2000). For Salmonella and Shigella species, samples were sent for serotypification to a laboratory (Asesores Especializados en Laboratorios S.A. de C.V.). 


\section{Crocodile attacks}

We used the Google search engine to search for any publications (scientific articles, reports, press articles) on attacks by crocodiles on humans in Mexican states of the Yucatan Peninsula (Quintana Roo, Campeche and Yucatan) in order to evaluate the number of attacks and changes in their frequency over time in the region. We also searched in the publications found for any reference to bacterial infection of a victim's wounds.

\section{Statistical analysis}

We used linear regressions to test the relationship between the number of bacteria in cloacal and oral cavities and crocodile total length. Results were considered significant at $\mathrm{p}<0.05$.

\section{RESULTS}

\section{Samples collected}

We captured 43 American crocodiles (4 hatchlings, 8 yearlings, 18 juveniles, 4 subadults and 9 adults) and 28 Morelet's crocodiles (4 hatchlings, 8 yearlings, 5 juveniles, 7 subadults and 4 adults). Thirtyfive American crocodiles were males (4 hatchlings, 8 yearlings, 16 juveniles, 3 subadults and 4 adults) and 8 were females (2 juveniles, 1 subadult and 5 adults). Of the Morelet's crocodiles captured, 22 were males (3 hatchlings, 7 yearlings, 2 juveniles, 6 subadults and 4 adults) and 6 were females (1 hatchling, 1 yearling, 3 juveniles and 1 subadult). We obtained oral samples from 38 American crocodiles (Table 1) and from all the 28 Morelet's crocodiles (Table 2). Five samples of juvenile American crocodiles were negative for bacteria after $72 \mathrm{~h}$ of incubation. We obtained cloacal samples from all American and Morelet's crocodiles captured (Tables 3 \& 4).

\section{American crocodile}

From the American crocodiles we isolated 32 bacterial species, of which $5(15.6 \%)$ were found in both oral and cloacal cavities and 17 (53.1\%) and 10 $(31.3 \%)$ were only found in the oral cavity and cloacal cavity, respectively. From oral samples of American crocodile we isolated 22 species of bacteria, of which 4 were determined to the genus level and 18 to the spe- cies level (Table 1). Of these bacteria 12 (54.5\%) were Gram-positive and 10 (45.5\%) were Gram-negative (Table 1). Arcanobacterium pyogenes and Aeromonas hydrophila were the most frequent isolates from oral samples and were isolated from 18 and 11 crocodiles, respectively (Table 1 ). The other bacteria were isolated from 1 to 6 crocodiles (Table 1). The number of bacteria species per crocodile ranged from 0 to 3 and was not correlated with crocodile total length $\left(\mathrm{r}^{2}=\right.$ 0.023, $\mathrm{p}=0.36, \mathrm{n}=28$ ). From cloacal samples of American crocodiles we found 15 species of bacteria, of which 2 were determined to the genus level and 13 to the species level (Table 3). Two (13.3\%) of these bacteria were Gram-positive and 13 (86.7\%) were Gramnegative (Table 3). Escherichia coli, A. hydrophila and Salmonella enterica subsp. arizonae were the most frequent bacteria in cloacal samples and were isolated from 29, 16 and 14 crocodiles, respectively (Table 3). The other bacteria were isolated from 1 to 8 crocodiles (Table 3). The number of bacteria species per crocodile ranged from 1 to 3 and was not correlated with crocodile total length $\left(r^{2}=0.058, p=0.12, n=43\right)$.

\section{Morelet's crocodile}

From the Morelet's crocodiles we isolated 29 different bacteria, of which 7 (24.1\%) were found in both oral and cloacal cavities and 15 (51.7\%) and 7 $(24.1 \%)$ were only found in the oral cavity and cloacal cavity, respectively. From oral samples we isolated 22 species of bacteria, of which 4 were determined to the genus level and 18 to the species level (Table 2). Eight $(36.4 \%)$ of these bacteria were Gram-positive and 14 (63.6\%) were Gram-negative (Table 2). The most frequent bacteria in the oral cavity of Morelet's crocodiles appeared to be Aeromonas hydrophila and Klebsiella pneumonia, which were isolated from 13 and 7 crocodiles, respectively (Table 2). All the other bacteria species were isolated from 1 to 4 crocodiles only (Table 2). The number of bacteria species per crocodile ranged from 1 to 3 and was not correlated with crocodile total length $\left(\mathrm{r}^{2}=0.163, \mathrm{p}=0.033, \mathrm{n}=\right.$ 28). From the cloacal samples, we determined a total of 14 species of bacteria (Table 4). All of these bacteria were Gram-negative and the most frequent bacteria found in cloacal samples of Morelet's crocodiles was Escherichia coli, which was detected in 18 $(64.3 \%)$ crocodiles (Table 4$)$. The other bacteria species were isolated from 1 to 7 crocodiles (Table 4). The number of bacteria species per crocodile ranged from 0 to 3 and was not correlated with crocodile total length $\left(\mathrm{r}^{2}=0.174, \mathrm{p}=0.027, \mathrm{n}=28\right)$. 
Table 1. Crocodylus acutus. Species of bacteria found in the oral cavity of wild American crocodiles and the number of swab samples from which each bacterial species was isolated, and the prevalence (\% in parentheses) of that bacterial species within each crocodile size class. FA: facultative anaerobic; A: aerobic; AN: anaerobic; ND: not determined

\begin{tabular}{|c|c|c|c|c|c|c|c|}
\hline Bacteria species & $\begin{array}{l}\text { Gram/ } \\
\text { type }\end{array}$ & $\begin{array}{l}\text { Hatchlings } \\
\quad(\mathrm{n}=4)\end{array}$ & $\begin{array}{l}\text { Yearlings } \\
(\mathrm{n}=7)\end{array}$ & $\begin{array}{l}\text { Juveniles } \\
(\mathrm{n}=19)\end{array}$ & $\begin{array}{l}\text { Sub-adults } \\
\quad(\mathrm{n}=3)\end{array}$ & $\begin{array}{l}\text { Adults } \\
(\mathrm{n}=5)\end{array}$ & $\begin{array}{c}\text { Total } \\
(\mathrm{n}=38)\end{array}$ \\
\hline Aeromonas hydrophila & $-/ \mathrm{FA}$ & $1(25.0)$ & $0(0.0)$ & $7(36.8)$ & $1(25.0)$ & $2(40.0)$ & $11(28.9)$ \\
\hline Arcanobacterium pyogenes & $+/ \mathrm{AN}$ & $4(100.0)$ & $6(85.7)$ & $6(31.6)$ & $1(25.0)$ & $1(20.0)$ & $18(47.4)$ \\
\hline Citrobacter freundii & $-/ \mathrm{FA}$ & $0(0.0)$ & $0(0.0)$ & $1(5.3)$ & $0(0.0)$ & $0(0.0)$ & $1(2.6)$ \\
\hline Corynebacterium sp. & $+/ \mathrm{FA}$ & $0(0.0)$ & $0(0.0)$ & $1(5.3)$ & $0(0.0)$ & $0(0.0)$ & $1(2.6)$ \\
\hline Enterococcus durans & $+/ \mathrm{A}$ & $0(0.0)$ & $0(0.0)$ & $1(5.3)$ & $1(25.0)$ & $0(0.0)$ & $2(5.3)$ \\
\hline Enterococcus faecium & $+/ \mathrm{FA}$ & $0(0.0)$ & $1(14.3)$ & $2(10.5)$ & $0(0.0)$ & $0(0.0)$ & $3(7.9)$ \\
\hline Escherichia coli & $-/ \mathrm{FA}$ & $0(0.0)$ & $1(14.3)$ & $0(0.0)$ & $0(0.0)$ & $0(0.0)$ & $1(2.6)$ \\
\hline Fusobacterium sp. & $-/ \mathrm{AN}$ & $0(0.0)$ & $1(14.3)$ & $0(0.0)$ & $0(0.0)$ & $0(0.0)$ & $1(2.6)$ \\
\hline Klebsiella pneumoniae & $-/ \mathrm{FA}$ & $0(0.0)$ & $0(0.0)$ & $0(0.0)$ & $1(25.0)$ & $0(0.0)$ & $1(2.6)$ \\
\hline Moraxella cuniculi & $-/ \mathrm{A}$ & $0(0.0)$ & $1(14.3)$ & $3(15.8)$ & $2(50.0)$ & $0(0.0)$ & $6(15.8)$ \\
\hline Moraxella catarrhalis & $-/ \mathrm{A}$ & $0(0.0)$ & $1(14.3)$ & $1(5.3)$ & $0(0.0)$ & $0(0.0)$ & $2(5.3)$ \\
\hline Pasteurella multocida & $-/ \mathrm{FA}$ & $0(0.0)$ & $0(0.0)$ & $1(5.3)$ & $0(0.0)$ & $0(0.0)$ & $1(2.6)$ \\
\hline Pseudomonas aeruginosa & $-/ \mathrm{A}$ & $0(0.0)$ & $1(14.3)$ & $2(10.5)$ & $0(0.0)$ & $0(0.0)$ & $3(7.9)$ \\
\hline Rhodococcus sp. & $+/ \mathrm{A}$ & $0(0.0)$ & $1(14.3)$ & $1(5.3)$ & $0(0.0)$ & $0(0.0)$ & $2(5.3)$ \\
\hline Serratia marcescens & $-/ \mathrm{A}$ & $2(50.0)$ & $0(0.0)$ & $0(0.0)$ & $0(0.0)$ & $4(80.0)$ & $6(15.8)$ \\
\hline Staphylococcus aureus & $+/ \mathrm{FA}$ & $1(25.0)$ & $0(0.0)$ & $0(0.0)$ & $1(25.0)$ & $4(80.0)$ & $6(15.8)$ \\
\hline Staphylococcus hyicus & $+/ \mathrm{FA}$ & $0(0.0)$ & $0(0.0)$ & $1(5.3)$ & $0(0.0)$ & $0(0.0)$ & $1(2.6)$ \\
\hline Streptococcus agalactiae & $+/ \mathrm{FA}$ & $0(0.0)$ & $1(14.3)$ & $4(21.1)$ & $1(25.0)$ & $0(0.0)$ & $6(15.8)$ \\
\hline Streptococcus intermedius & $+/ \mathrm{AN}$ & $0(0.0)$ & $0(0.0)$ & $1(5.3)$ & $0(0.0)$ & $0(0.0)$ & $1(2.6)$ \\
\hline Streptococcus pneumoniae & $+/ \mathrm{FA}$ & $0(0.0)$ & $0(0.0)$ & $1(5.3)$ & $0(0.0)$ & $0(0.0)$ & $1(2.6)$ \\
\hline Streptococcus pyogenes & $+/ \mathrm{A}$ & $0(0.0)$ & $0(0.0)$ & $0(0.0)$ & $0(0.0)$ & $1(20.0)$ & $1(2.6)$ \\
\hline Streptococcus sp. & $+/ \mathrm{ND}$ & $0(0.0)$ & $1(14.3)$ & $0(0.0)$ & $0(0.0)$ & $0(0.0)$ & $1(2.6)$ \\
\hline Total species/size class & & 4 & 10 & 15 & 7 & 5 & 22 \\
\hline $\begin{array}{l}\text { Mean }( \pm \mathrm{SD}) \text { species } \\
\text { per crocodile }\end{array}$ & & $2.0 \pm 0.0$ & $2.1 \pm 1.1$ & $1.7 \pm 1.1$ & $2.0 \pm 1.4$ & $2.4 \pm 0.6$ & $2.0 \pm 1.0$ \\
\hline
\end{tabular}

Table 2. Crocodylus moreletii. Species of bacteria found in the oral cavity of wild Morelet's crocodiles and the number of swab samples from which each bacterial species was isolated, and the prevalence (\% in parentheses) of that bacterial species within each crocodile size class. FA: facultative anaerobic; A: aerobic; AN: anaerobic

\begin{tabular}{|c|c|c|c|c|c|c|c|}
\hline Bacteria species & $\begin{array}{l}\text { Gram/ } \\
\text { type }\end{array}$ & $\begin{array}{l}\text { Hatchlings } \\
(\mathrm{n}=4)\end{array}$ & $\begin{array}{l}\text { Yearlings } \\
(\mathrm{n}=8)\end{array}$ & $\begin{array}{l}\text { Juveniles } \\
\qquad(\mathrm{n}=5)\end{array}$ & $\begin{array}{l}\text { Sub-adults } \\
\quad(\mathrm{n}=7)\end{array}$ & $\begin{array}{l}\text { Adults } \\
(\mathrm{n}=4)\end{array}$ & $\begin{array}{c}\text { Total } \\
(\mathrm{n}=28)\end{array}$ \\
\hline Aerococcus viridans & $+/ \mathrm{A}$ & $0(0.0)$ & $0(0.0)$ & $1(20.0)$ & $2(28.6)$ & $1(25.0)$ & $4(14.3)$ \\
\hline Aeromonas hydrophila & $-/ F A$ & $0(0.0)$ & $6(75.0)$ & $1(20.0)$ & $4(57.1)$ & $2(50.0)$ & $13(46.4)$ \\
\hline Arcanobacterium pyogenes & $+/ \mathrm{AN}$ & $0(0.0)$ & $1(12.5)$ & $0(0.0)$ & $2(28.6)$ & $0(0.0)$ & $3(10.7)$ \\
\hline Citrobacter freundii & $-/ \mathrm{FA}$ & $0(0.0)$ & $1(12.5)$ & $1(20.0)$ & $2(28.6)$ & $0(0.0)$ & $4(14.3)$ \\
\hline Escherichia coli & $-/ F A$ & $1(25.0)$ & $0(0.0)$ & $0(0.0)$ & $0(0.0)$ & $0(0.0)$ & $1(3.6)$ \\
\hline Fusobacterium necrophorum & $-/ \mathrm{AN}$ & $0(0.0)$ & $1(12.5)$ & $0(0.0)$ & $0(0.0)$ & $0(0.0)$ & $1(3.6)$ \\
\hline Fusobacterium sp. & $-/ \mathrm{AN}$ & $0(0.0)$ & $0(0.0)$ & $0(0.0)$ & $0(0.0)$ & $1(25.0)$ & $1(3.6)$ \\
\hline Klebsiella pneumoniae & $-/ \mathrm{FA}$ & $2(50.0)$ & $1(12.5)$ & $0(0.0)$ & $2(28.6)$ & $2(50.0)$ & $7(25.0)$ \\
\hline Kluyvera sp. & $-/ \mathrm{FA}$ & $0(0.0)$ & $0(0.0)$ & $1(20.0)$ & $0(0.0)$ & $0(0.0)$ & $1(3.6)$ \\
\hline Listeria monocytogenes & $+/ \mathrm{FA}$ & $0(0.0)$ & $1(12.5)$ & $0(0.0)$ & $1(14.3)$ & $0(0.0)$ & $2(7.1)$ \\
\hline Obesumbacterium proteus & $-/ \mathrm{FA}$ & $0(0.0)$ & $0(0.0)$ & $1(20.0)$ & $1(14.3)$ & $0(0.0)$ & $2(7.1)$ \\
\hline Pantoea agglomerans & $-/ F A$ & $0(0.0)$ & $0(0.0)$ & $1(20.0)$ & $0(0.0)$ & $0(0.0)$ & $1(3.6)$ \\
\hline Proteus vulgaris & $-/ \mathrm{FA}$ & $0(0.0)$ & $1(12.5)$ & $0(0.0)$ & $0(0.0)$ & $0(0.0)$ & $1(3.6)$ \\
\hline Pseudomonas fluorescens & $-/ \mathrm{A}$ & $1(25.0)$ & $0(0.0)$ & $1(20.0)$ & $0(0.0)$ & $0(0.0)$ & $2(7.1)$ \\
\hline Rhodococcus equi & $+/ \mathrm{A}$ & $0(0.0)$ & $0(0.0)$ & $0(0.0)$ & $1(14.3)$ & $0(0.0)$ & $1(3.6)$ \\
\hline Salmonella arizonae & $-/ \mathrm{FA}$ & $0(0.0)$ & $0(0.0)$ & $0(0.0)$ & $1(14.3)$ & $1(25.0)$ & $2(7.1)$ \\
\hline Serratia rubidaea & $-/ \mathrm{FA}$ & $0(0.0)$ & $1(12.5)$ & $0(0.0)$ & $1(14.3)$ & $1(25.0)$ & $3(10.7)$ \\
\hline Shigella sp. & -/FA & $0(0.0)$ & $0(0.0)$ & $0(0.0)$ & $1(14.3)$ & $0(0.0)$ & $1(3.6)$ \\
\hline Staphylococcus aureus & $+/ \mathrm{FA}$ & $0(0.0)$ & $0(0.0)$ & $1(20.0)$ & $0(0.0)$ & $0(0.0)$ & $1(3.6)$ \\
\hline Staphylococcus sp. & $+/ \mathrm{FA}$ & $0(0.0)$ & $3(37.5)$ & $0(0.0)$ & $0(0.0)$ & $0(0.0)$ & $3(10.7)$ \\
\hline Streptococcus agalactiae & $+/ \mathrm{FA}$ & $0(0.0)$ & $0(0.0)$ & $0(0.0)$ & $1(14.3)$ & $1(25.0)$ & $2(7.1)$ \\
\hline Streptococcus pneumoniae & $+/ \mathrm{FA}$ & $0(0.0)$ & $2(25.0)$ & $0(0.0)$ & $0(0.0)$ & $0(0.0)$ & $2(7.1)$ \\
\hline Total species/size class & & 3 & 10 & 8 & 12 & 7 & 22 \\
\hline $\begin{array}{l}\text { Mean }( \pm \mathrm{SD}) \text { species } \\
\text { per crocodile }\end{array}$ & & $1.0 \pm 0.0$ & $2.3 \pm 0.5$ & $1.6 \pm 0.6$ & $2.7 \pm 0.5$ & $2.3 \pm 0.8$ & $2.1 \pm 0.8$ \\
\hline
\end{tabular}


Table 3. Crocodylus acutus. Species of bacteria found in the cloacal cavity of wild American crocodiles and the number of swab samples from which each bacterial species was isolated, and the prevalence (\% in parentheses) of that bacterial species within each crocodile size class. FA: facultative anaerobic; A: aerobic

\begin{tabular}{|c|c|c|c|c|c|c|c|}
\hline Bacteria species & $\begin{array}{l}\text { Gram/ } \\
\text { type }\end{array}$ & $\begin{array}{l}\text { Hatchlings } \\
\quad(\mathrm{n}=4)\end{array}$ & $\begin{array}{l}\text { Yearlings } \\
(\mathrm{n}=8)\end{array}$ & $\begin{array}{l}\text { Juveniles } \\
(\mathrm{n}=18)\end{array}$ & $\begin{array}{l}\text { Sub-adults } \\
(\mathrm{n}=4)\end{array}$ & $\begin{array}{l}\text { Adults } \\
(\mathrm{n}=9)\end{array}$ & $\begin{array}{c}\text { Total } \\
(\mathrm{n}=43)\end{array}$ \\
\hline Aeromonas hydrophila & $-/ \mathrm{FA}$ & $2(50.0)$ & $3(37.5)$ & $9(50.0)$ & $0(0.0)$ & $2(22.2)$ & $16(37.2)$ \\
\hline Alcaligenes faecalis & $-/ \mathrm{A}$ & $0(0.0)$ & $0(0.0)$ & $2(11.1)$ & $0(0.0)$ & $0(0.0)$ & $2(4.7)$ \\
\hline Citrobacter diversus & $-/ \mathrm{FA}$ & $1(25.0)$ & $3(37.5)$ & $2(11.1)$ & $0(0.0)$ & $2(22.2)$ & $8(18.6)$ \\
\hline Citrobacter freundii & $-/ \mathrm{FA}$ & $0(0.0)$ & $1(12.5)$ & $3(16.7)$ & $0(0.0)$ & $0(0.0)$ & $4(9.3)$ \\
\hline Escherichia coli & $-/ \mathrm{FA}$ & $1(25.0)$ & $6(75.0)$ & $13(72.2)$ & $3(75.0)$ & $6(66.7)$ & $29(67.4)$ \\
\hline Enterobacter cloacae & $-/ \mathrm{FA}$ & $1(25.0)$ & $0(0.0)$ & $2(11.1)$ & $2(50.0)$ & $1(11.1)$ & $6(14.0)$ \\
\hline Enterococcus faecalis & $+/ \mathrm{FA}$ & $0(0.0)$ & $1(12.5)$ & $5(27.8)$ & $0(0.0)$ & $0(0.0)$ & $6(14.0)$ \\
\hline Edwardsiella tarda & $-/ \mathrm{FA}$ & $2(50.0)$ & $0(0.0)$ & $1(5.6)$ & $0(0.0)$ & $2(22.2)$ & $5(11.6)$ \\
\hline Klebsiella pneumoniae & $-/ \mathrm{FA}$ & $0(0.0)$ & $0(0.0)$ & $0(0.0)$ & $1(25.0)$ & $0(0.0)$ & $1(2.3)$ \\
\hline Klebsiella sp. & $-/ \mathrm{FA}$ & $0(0.0)$ & $1(12.5)$ & $1(5.6)$ & $0(0.0)$ & $0(0.0)$ & $2(4.7)$ \\
\hline Proteus mirabilis & $-/ \mathrm{FA}$ & $0(0.0)$ & $1(12.5)$ & $0(0.0)$ & $0(0.0)$ & $0(0.0)$ & $1(2.3)$ \\
\hline Proteus vulgaris & $-/ \mathrm{FA}$ & $1(25.0)$ & $0(0.0)$ & $5(27.8)$ & $0(0.0)$ & $2(22.2)$ & $8(18.6)$ \\
\hline Rhodococcus sp. & $+/ \mathrm{FA}$ & $0(0.0)$ & $2(25.0)$ & $2(11.1)$ & $1(25.0)$ & $0(0.0)$ & $5(11.6)$ \\
\hline Salmonella arizonae & $-/ \mathrm{FA}$ & $1(25.0)$ & $3(37.5)$ & $5(27.8)$ & $1(25.0)$ & $4(44.4)$ & $14(32.6)$ \\
\hline Shigella sonnei & $-/ \mathrm{FA}$ & $0(0.0)$ & $0(0.0)$ & $0(0.0)$ & $0(0.0)$ & 1 (11.1) & $1(2.3)$ \\
\hline Total species/size class & & 7 & 9 & 12 & 5 & 8 & 15 \\
\hline $\begin{array}{l}\text { Mean }( \pm \mathrm{SD}) \text { species } \\
\text { per crocodile }\end{array}$ & & $2.3 \pm 0.5$ & $2.6 \pm 0.5$ & $2.8 \pm 0.4$ & $2.0 \pm 0.8$ & $2.2 \pm 0.8$ & $2.5 \pm 0.6$ \\
\hline
\end{tabular}

Table 4. Crocodylus moreletii. Species of bacteria found in the cloacal cavity of wild Morelet's crocodiles and the number of swab samples from which each bacterial species was isolated, and the prevalence (\% in parentheses) of that bacterial species within each crocodile size class. FA: facultative anaerobic; AF: aerobic facultative

\begin{tabular}{|c|c|c|c|c|c|c|c|}
\hline Bacteria species & $\begin{array}{l}\text { Gram/ } \\
\text { type }\end{array}$ & $\begin{array}{l}\text { Hatchlings } \\
\quad(\mathrm{n}=4)\end{array}$ & $\begin{array}{l}\text { Yearlings } \\
(\mathrm{n}=8)\end{array}$ & $\begin{array}{l}\text { Juveniles } \\
\quad(\mathrm{n}=5)\end{array}$ & $\begin{array}{l}\text { Sub-adults } \\
(\mathrm{n}=7)\end{array}$ & $\begin{array}{l}\text { Adults } \\
(\mathrm{n}=4)\end{array}$ & $\begin{array}{c}\text { Total } \\
(\mathrm{n}=28)\end{array}$ \\
\hline Aeromomas hydrophila & $-/ \mathrm{FA}$ & $0(0.0)$ & $0(0.0)$ & $0(0.0)$ & $0(0.0)$ & $1(25.0)$ & $1(3.6)$ \\
\hline Citrobacter diversus & $-/ \mathrm{FA}$ & $0(0.0)$ & $1(12.5)$ & $0(0.0)$ & $1(14.3)$ & $0(0.0)$ & $2(7.1)$ \\
\hline Edwarsiella tarda & $-/ \mathrm{FA}$ & $0(0.0)$ & $1(12.5)$ & $1(20.0)$ & $0(0.0)$ & $0(0.0)$ & $2(7.1)$ \\
\hline Enterobacter cloacae & $-/ \mathrm{FA}$ & $0(0.0)$ & $2(25.0)$ & $2(40.0)$ & $0(0.0)$ & $1(25.0)$ & $5(17.9)$ \\
\hline Enterobacter gergoviae & $-/ \mathrm{FA}$ & $0(0.0)$ & $0(0.0)$ & $0(0.0)$ & $0(0.0)$ & $1(25.0)$ & $1(3.6)$ \\
\hline Escherichia coli & $-/ \mathrm{FA}$ & $0(0.0)$ & $8(100)$ & $2(40.0)$ & $6(85.7)$ & $2(50.0)$ & $18(64.3)$ \\
\hline Hafnia alvei & $-/ \mathrm{AF}$ & $0(0.0)$ & $0(0.0)$ & $1(20.0)$ & $0(0.0)$ & $0(0.0)$ & $1(3.6)$ \\
\hline Klebsiella pneumoniae & $-/ \mathrm{FA}$ & $0(0.0)$ & $2(25.0)$ & $0(0.0)$ & $2(28.6)$ & $2(50.0)$ & $6(21.4)$ \\
\hline Pantoea agglomerans & $-/ \mathrm{FA}$ & $0(0.0)$ & $0(0.0)$ & $1(20.0)$ & $0(0.0)$ & $1(25.0)$ & $2(7.1)$ \\
\hline Proteus vulgaris & $-/ \mathrm{FA}$ & $0(0.0)$ & $0(0.0)$ & $0(0.0)$ & $1(14.3)$ & $0(0.0)$ & $1(3.6)$ \\
\hline Salmonellla arizonae & $-/ \mathrm{FA}$ & $0(0.0)$ & $2(25.0)$ & $1(20.0)$ & $3(42.9)$ & $1(25.0)$ & $7(25.0)$ \\
\hline Salmonella typhi & $-/ \mathrm{FA}$ & $0(0.0)$ & $1(12.5)$ & $0(0.0)$ & $0(0.0)$ & $0(0.0)$ & $1(3.6)$ \\
\hline Serratia rubidaea & $-/ \mathrm{FA}$ & $0(0.0)$ & $0(0.0)$ & $1(20.0)$ & $2(28.6)$ & $1(25.0)$ & $4(14.3)$ \\
\hline Yersinia enterocolitica & $-/ \mathrm{FA}$ & $0(0.0)$ & $1(12.5)$ & $0(0.0)$ & $2(28.6)$ & $0(0.0)$ & $3(10.7)$ \\
\hline Total species/size class & & 0 & 8 & 7 & 7 & 8 & 14 \\
\hline $\begin{array}{l}\text { Mean }( \pm \text { SD) species } \\
\text { per crocodile }\end{array}$ & & 0 & $2.3 \pm 0.7$ & $1.8 \pm 0.8$ & $2.4 \pm 0.5$ & $2.5 \pm 1.0$ & $2.3 \pm 0.7$ \\
\hline
\end{tabular}

\section{Interspecific differences}

In total, we isolated 47 species of bacteria from all samples, distributed within 28 genera and 14 families (Table 5). Species of the family Enterobacteriaceae represented $51.1 \%$ of the species. Fourteen species $(29.8 \%)$ were detected in both croco- dile species and 18 (38.3\%) and 15 (31.9\%) species were only detected in American crocodiles and Morelet's crocodiles, respectively. We found 35 species (23 genera, 13 families) of bacteria in all oral samples, of which 9 species (25.8\%) were detected in both crocodile species. Thirteen species $(37.1 \%)$ were only detected in Morelet's crocodiles 
Table 5. Crocodylus acutus and C. moreletii. All bacteria species found in American and Morelet's crocodiles by capture location indicating the cavity in which each bacterium was isolated (O: oral; $\mathrm{C}$ : cloaca; -: not detected)

\begin{tabular}{|c|c|c|c|c|}
\hline Family & Species & $\begin{array}{l}\text { Río Hondo } \\
\text { (C. moreletii) }\end{array}$ & $\begin{array}{l}\text { Cozumel } \\
\text { (C. acutus) }\end{array}$ & $\begin{array}{c}\text { Banco Chinchorro } \\
\text { (C. acutus) }\end{array}$ \\
\hline Actinomycetaceae & Arcanobacterium pyogenes & $\mathrm{O}$ & $\mathrm{O}$ & $\mathrm{O}$ \\
\hline Aerococcaceae & Aerococcus viridans & $\mathrm{O}$ & - & - \\
\hline Aeromonadaceae & Aeromomas hydrophila & $\mathrm{O}, \mathrm{C}$ & $\mathrm{O}, \mathrm{C}$ & $\mathrm{O}, \mathrm{C}$ \\
\hline Alcaligenaceae & Alcaligenes faecalis & - & $\mathrm{C}$ & - \\
\hline Corynebacteriaceae & Corynebacterium sp. & - & $\mathrm{O}$ & - \\
\hline \multirow[t]{24}{*}{ Enterobacteriaceae } & Citrobacter diversus & $\mathrm{C}$ & $\mathrm{C}$ & $\mathrm{C}$ \\
\hline & Citrobacter freundii & $\mathrm{O}$ & $\mathrm{O}, \mathrm{C}$ & - \\
\hline & Edwarsiella tarda & $\mathrm{C}$ & $\mathrm{C}$ & $\mathrm{C}$ \\
\hline & Enterobacter cloacae & $\mathrm{C}$ & $\mathrm{C}$ & $\mathrm{C}$ \\
\hline & Enterobacter gergoviae & $\mathrm{C}$ & - & - \\
\hline & Enterococcus durans & - & $\mathrm{O}$ & - \\
\hline & Enterococcus faecalis & - & $\mathrm{C}$ & - \\
\hline & Enterococcus faecium & - & $\mathrm{O}$ & - \\
\hline & Escherichia coli & $\mathrm{O}, \mathrm{C}$ & $\mathrm{O}, \mathrm{C}$ & $\mathrm{C}$ \\
\hline & Hafnia alvei & $\mathrm{C}$ & - & - \\
\hline & Klebsiella pneumoniae & $\mathrm{O}, \mathrm{C}$ & $\mathrm{O}, \mathrm{C}$ & - \\
\hline & Klebsiella sp. & - & $\mathrm{C}$ & - \\
\hline & Kluyvera sp. & $\mathrm{O}$ & - & - \\
\hline & Obesumbacterium proteus & $\mathrm{O}$ & - & - \\
\hline & Pantoea agglomerans & $\mathrm{O}, \mathrm{C}$ & - & - \\
\hline & Proteus mirabilis & - & $\mathrm{C}$ & - \\
\hline & Proteus vulgaris & $\mathrm{O}, \mathrm{C}$ & $\mathrm{C}$ & $\mathrm{C}$ \\
\hline & Salmonella enterica arizonae & $\mathrm{O}, \mathrm{C}$ & $\mathrm{C}$ & $\mathrm{C}$ \\
\hline & Salmonella enterica enterica ser. Typhi & $\mathrm{C}$ & - & - \\
\hline & Serratia marcescens & - & - & $\mathrm{O}$ \\
\hline & Serratia rubidaea & $\mathrm{O}, \mathrm{C}$ & - & - \\
\hline & Shigella sonnei & - & - & $\mathrm{C}$ \\
\hline & Shigella sp. & $\mathrm{O}$ & - & - \\
\hline & Yersinia enterocolitica & $\mathrm{C}$ & - & - \\
\hline \multirow[t]{2}{*}{ Fusobacteriaceae } & Fusobacterium necrophorum & $\mathrm{O}$ & - & - \\
\hline & Fusobacterium sp. & $\mathrm{O}$ & $\mathrm{O}$ & - \\
\hline Listeriaceae & Listeria monocytogenes & $\mathrm{O}$ & - & - \\
\hline \multirow[t]{2}{*}{ Moraxellaceae } & Moraxella catarrhalis & - & $\mathrm{O}$ & - \\
\hline & Moraxella cuniculi & - & $\mathrm{O}$ & - \\
\hline \multirow[t]{2}{*}{ Nocardiaceae } & Rhodococcus equi & $\mathrm{O}$ & - & - \\
\hline & Rhodococcus sp. & - & $\mathrm{O}, \mathrm{C}$ & - \\
\hline Pasteurellaceae & Pasteurella multocida & - & $\mathrm{O}$ & - \\
\hline \multirow[t]{2}{*}{ Pseudomodaceae } & Pseudomonas aeruginosa & - & $\mathrm{O}$ & - \\
\hline & Pseudomonas fluorescens & $\mathrm{O}$ & - & - \\
\hline \multirow[t]{3}{*}{ Staphylococcaceae } & Staphylococcus aureus & $\mathrm{O}$ & - & $\mathrm{O}$ \\
\hline & Staphylococcus hyicus & - & $\mathrm{O}$ & - \\
\hline & Staphylococcus sp. & $\mathrm{O}$ & - & - \\
\hline \multirow[t]{5}{*}{ Streptococcaceae } & Streptococcus agalactiae & $\mathrm{O}$ & $\mathrm{O}$ & - \\
\hline & Streptococcus intermedius & - & $\mathrm{O}$ & - \\
\hline & Streptococcus pneumoniae & $\mathrm{O}$ & $\mathrm{O}$ & - \\
\hline & Streptococcus pyogenes & - & - & $\mathrm{O}$ \\
\hline & Streptococcus sp. & - & $\mathrm{O}$ & - \\
\hline
\end{tabular}

and another 13 species (37.1\%) were only detected in American crocodiles. From all cloacal samples, we found a total of 21 species (16 genera, 5 families) of bacteria, of which 8 species $(38.1 \%)$ were detected in both crocodile species, and $6(28.6 \%)$ and $7(33.3 \%)$ species were only detected in Morelet's crocodiles and American crocodiles, respectively.

\section{Crocodile attacks}

We found 12 publications that referred to attacks by crocodiles on humans in the Yucatan Peninsula. We also knew of 2 cases of attacks by American crocodiles on fishers in Banco Chinchorro during our field work (P. Charruau pers. obs.). From these publications and observations, we determined that a mini- 
mum of 24 attacks occurred in the Yucatan Peninsula (21 in Quintana Roo, 2 in Campeche and 1 in Yucatan) from 1992 to 2011. Two were fatal (1 in Campeche and 1 in Quintana Roo) and evidence indicated that Crocodylus moreletii was responsible. Both species (C. acutus and C. moreletii) were involved in the attacks. Most of the attacks occurred when victims were in the water fishing, spear-fishing or swimming, and in 4 cases victims were under the influence of alcohol. Among the victims only 1 woman $(4.2 \%)$ was involved. The number of attacks in Quintana Roo shows an increase of attacks during the last 6 yr, after almost a decade without incidents (Fig. 2). Information about injuries of victims is available in most of the press articles; injuries consisted of bites on various parts of the body (shoulders, hands, arms, foot, thorax, pelvis, back, legs, neck and head), lung perforation, partial loss of a foot and death (in 2 cases). However, the press articles do not present information about infection and bacteria involved.

\section{DISCUSSION}

Few studies on bacterial flora in cloacal and oral cavities of wild crocodilians have been done and, as far as we know, only 5 crocodilian species have been examined in wild conditions: Alligator mississippiensis (Flandry et al. 1989, Johnston et al. 2010), Crocodylus acutus (Cupul-Magaña et al. 2005), C. niloticus (Lovely \& Leslie 2008), C. porosus (Anderson 1999) and C. johnstoni (Anderson 1999). To our knowledge, the present study is the first record of bacterial flora in oral and cloacal cavities of wild C. moreletii. The present study is the second to investigate oral flora and the first on cloacal flora for $C$. acutus in the wild. Before this study, Cupul-Magaña et al. (2005) examined the oral flora of wild C. acutus, and found 10

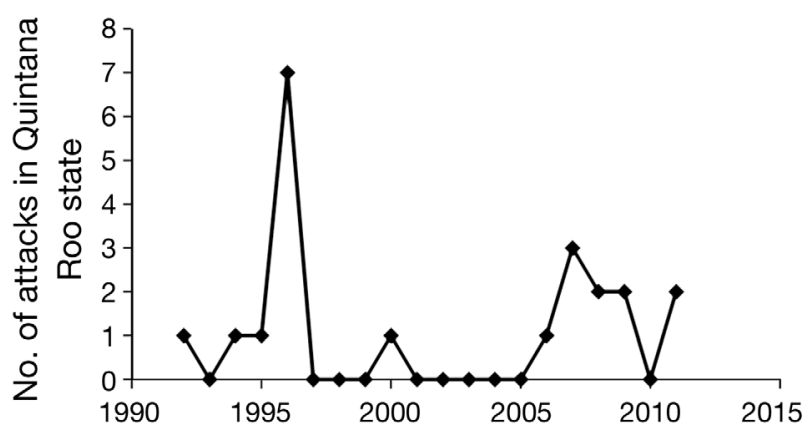

Fig. 2. Crocodylus acutus and C. moreletii. Number of attacks by American and Morelet's crocodiles on humans in Quintana Roo State, Mexico, from 1992 to 2011 bacteria species of which 5 were also found in this study either in the cloacal or oral cavity (Aeromonas hydrophila, Citrobacter freundii, C. diversus, Escherichia coli and Klebsiella pneumoniae).

The genera Aeromonas, Citrobacter, Corynebacterium, Enterococcus, Escherichia, Fusobacterium, Klebsiella, Moraxella, Pasteurella, Proteus, Pseudomonas, Salmonella, Serratia, Shigella, Staphylococcus and Streptococcus have been already identified in the oral cavity of crocodilians (Doering et al. 1971, Gorden et al. 1979, Flandry et al. 1989, Anderson 1999, Cupul-Magaña et al. 2005, Silva et al. 2009). To our knowledge, the present study documents for the first time the occurrence of the genera Aerococcus, Arcanobacterium, Kluyvera, Listeria, Obesumbacterium, Pantoea and Rhodococcus in the oral cavity of wild crocodilians. It is also the first record of occurrence of the following species in the oral cavity of crocodilians: Aerococcus viridians, Arcanobacterium pyogenes, Enterococcus durans, E. faecium, Fusobacterium necrophorum, Listeria monocytogenes, Moraxella cuniculi, M. catarrhalis, Obesumbacterium proteus, Pantoea agglomerans, Pasteurella multocida, Rhodococcus equi, Salmonella enterica arizonae, Serratia rubidaea, Staphylococcus aureus, S. hyicus, Streptococcus agalactiae, $S$. intermedius and S. pneumoniae.

The genera Aeromonas, Citrobacter, Edwarsiella, Enterobacter, Enterococcus, Escherichia, Hafnia, Klebsiella, Pantoea, Proteus, Salmonella, Serratia and Yersinia have been already identified in cloaca of crocodilians (White et al. 1973, Madsen et al. 1998, Anderson 1999, Lovely \& Leslie 2008, Johnston et al. 2010). To our knowledge, this is the first report of the genera Alcaligenes and Rhodococcus in the cloaca. The following species appear to be new reports for the cloacal flora of crocodilians: Alcaligenes faecalis, Citrobacter diversus, Enterobacter gergoviae, Pantoea agglomerans, Salmonella enterica enterica serotype Typhi, Serratia rubidaea and Yersinia enterocolitica.

Crocodilians are very resistant to disease with a great capacity for healing, principally owing to their high level of serum antimicrobial activity (Merchant \& Britton 2006, Merchant et al. 2006). Huchzermeyer (2003) report that few bacteria cause specific diseases in crocodiles and even fewer are crocodilespecific, but many bacteria can cause non-specific septicemia. As all crocodiles captured during the present study were apparently healthy, we assume that all bacteria species found in the present study are part of the normal flora of Crocodylus acutus and C. moreletii. However, several of the bacteria iso- 
lated could be pathogens of crocodilians when the host is subject to stress, particularly in captive conditions (Huchzermeyer 2003). Bacteria species carried by $C$. acutus and/or C. moreletii in the Yucatan Peninsula and isolated from cases of crocodilian septicemias are: Aeromonas hydrophila, Arcanobacterium pyogenes, Citrobacter freundii, Corynebacterium sp., Edwardsiella tarda, Escherichia coli, Klebsiella sp., Pantoea agglomerans, Pasteurella multocida, Proteus sp., Pseudomonas aeruginosa, Serratia marcescens, Staphylococcus aureus, Staphylococcus sp. and Streptococcus sp. (Huchzermeyer 2003). Moreover, Streptococcus agalactiae has been reported to cause necrotizing fasciitis in captive juvenile Crocodylus porosus (Bishop et al. 2007).

A large number of Salmonella serovars have been isolated from crocodiles (Huchzermeyer 2003) and are part of the normal intestinal tract flora of crocodilians. However, Salmonella can cause enteritis and septicemia in individuals subjected to severe stress conditions (Huchzermeyer 2003). In the present study, we identified 2 subspecies of Salmonella enterica ( $S$. enterica subsp. arizonae in the oral and cloacal cavities of Crocodylus moreletii and in the cloacae of $C$. acutus, and $S$. enterica subsp. enterica serotype Typhi from the cloacal cavity of C. moreletii), which in certain stressful conditions could cause enteritis and septicemia in C. moreletii and C. acutus.

Cloacal flora can also be responsible for infections of the egg shell, egg shell membrane and yolk during egg laying (Huchzermeyer 2003). Peucker et al. (2005) studied bacteria present in shell and yolk of farmed Australian freshwater crocodile Crocodylus johnstoni eggs and among the bacteria they found, 13 have been identified in $C$. acutus and/or $C$. moreletii in the present study: Aeromonas hydrophila, Alcaligenes faecalis, Citrobacter freundii, Edwardsiella tarda, Enterobacter cloacae, Escherichia coli, Klebsiella sp., Pantoea agglomerans, Proteus vulgaris, Pseudomonas aeruginosa, P. fluorescens, Salmonella arizonae and Serratia marcescens. Schumacher \& Cardeilhac (1990) also found Enterobacter cloacae, Citrobacter sp., Proteus sp. and Pseudomonas aeruginosa in lesions of infected eggs, and Thomas et al. (2001) isolated several Salmonella serotypes from the egg shell or yolk of Crocodylus porosus. Bacterial egg infection is a cause of egg mortality and can also lead to yolk-sac infections and omphalitis in hatchlings, which can result in their death (Huchzermeyer 2003). This information is important if we consider that $C$. acutus in Banco Chinchorro show a low nest number and a reduced clutch size (Charruau et al. 2010). In this condition, bacterial infection of eggs could be a threat to this crocodile's conservation, and an assessment of bacteria present on C. acutus egg shell in Banco Chinchorro should therefore be made.

Bacteria species carried by crocodiles can also be transmitted to humans. The transmission of bacteria from crocodiles to humans can occur in 3 major ways: by consuming crocodile meat (Suárez et al. 2000, Magnino et al. 2009), from bites during crocodile attacks (Caldicott et al. 2005, Gruen 2009, Wamisho et al. 2009) or during activities in 'crocodile waters' (Johnston et al. 2010). Wounds from crocodile bites generally become infected by bacteria present in the oral cavity of crocodiles (Caldicott et al. 2005, Wamisho et al. 2009). Bacteria involved in wound infections after crocodilian attacks include Aeromonas hydrophila, Burkholderia pseudomallei, Pseudomonas spp., Serratia spp., Citrobacter diversus, Enterococcus spp., Clostridium spp. and Pantoea agglomerans (Raynor et al. 1983, Flandry et al. 1989, Mekisic \& Wardill 1992). Most of these species and genera have been identified in the present study in oral and/or cloacal cavity of American or Morelet's crocodiles. Moreover, among bacteria identified in oral cavity of crocodiles in this study, Aeromonas hydrophila, Corynebacterium sp., Citrobacter freundii, Enterococcus durans, E. faecium, Escherichia coli, Klebsiella pneumonia, Pantoea agglomerans, Proteus vulgaris, Salmonella enterica arizonae, Serratia marcescens, S. rubidaea, Fusobacterium necrophorum, Fusibacterium sp., Moraxella catarrahalis, M. cuniculi, Rhodococcus sp., Pasteurella multocida, Pseudomonas aeruginosa, P. fluorescens, Staphylococcus aureus, S. hyicus, Staphylococcus sp., Streptococcus agalactiae, S. intermedius, S. pyogenes and Streptococcus sp. have been reported in infected wounds caused by animal or human bites (Murphey et al. 1992, Blaylock 1999, Goldstein et al. 2002, Kunimoto et al. 2004, Jofré Morales et al. 2006, Brook 2009).

In the present study we found 24 reported cases of attacks by crocodiles on humans in the Yucatan Peninsula, most of them (87.5\%) occurring in Quintana Roo and in the city of Cancun, which are the state and city in the peninsula, respectively, most frequented by tourists. Both crocodile species (Crocodylus acutus and $C$. moreletii) were involved in the attacks and C. moreletii was involved in 2 cases of fatal attacks. Attacks resulted in a variety of wounds on the victim from bites to different parts of the body to lung perforation, limb amputation and death. Bacteria found in oral cavities of crocodiles could cause 
infections and septicemia of wounds reported in the attacks. However, no publications reporting the attacks present information about wound infection and bacteria involved. Furthermore, the number of attacks in Quintana Roo seems to have increased since 2005 (Fig. 2). In the country of Belize, adjacent to Quintana Roo, several studies have also reported an increase in the number of attacks on humans by crocodiles (Garel et al. 2005). These attacks involved C. moreletii and no case of attack by C. acutus was reported (Marlin et al. 1995, Finger et al. 2002, Garel et al. 2005). The principal factors of this increase in crocodile-human interactions in Quintana Roo and Belize are the increase in human and crocodile populations, the destruction and fragmentation of crocodile habitat, the deliberate and unintentional feeding of crocodiles in human-populated areas, the increased efforts in recording crocodile attacks, drought causing loss of water in lagoons that increase the density of crocodiles and the economic situation in Mexico that increases illegal and subsistence fishing, thereby increasing the probability of crocodile encounters (Lazcano-Barrero 1996, Finger et al. 2002, Garel et al. 2005).

Farmed crocodilian meat harvested for human consumption often shows bacterial contamination, which principally occurs during slaughter and dressing procedures (Revol 1995, Rickard et al. 1995, Thomas et al. 2001, Magnino et al. 2009). Microbiological analyses of meat of several farmed crocodilian species have shown the presence of the bacteria genera Salmonella, Staphylococcus, Flavobacterium, Pseudomonas, Acinobacter, Enterobacter, Moraxella, Micrococcus, Streptococcus and Escherichia (Madsen 1993, Rickard et al. 1995, Hoffmann \& Romanelli 1998, Thomas et al. 2001). Furthermore, infection by Bacillus cereus after black caiman Melanosuchus niger meat consumption has been reported in the Amazonas (Suárez et al. 2000). A case of salmonellosis in South Africa has also been reported in a man who consumed crocodile meat infected by Salmonella enterica diarizonae (Narayana et al. 2008). Cases of salmonellosis in humans often occurred after direct or indirect contact with reptiles (Mermin et al. 2004). Although crocodiles are protected and their hunt has been banned since 1970 in Mexico, opportunistic killings occur occasionally in the vicinity of human settlements (Cedeño-Vázquez et al. 2006) and meat consumption of poached individuals could cause infection. Furthermore, during crocodile population surveys in the Yucatan Peninsula, we occasionally observed children who kept hatchling crocodiles as pets, and they could contract salmonel- losis by contact with those crocodiles. We especially warn about the presence of $S$. enterica enterica ser. Typhi in the cloaca of Morelet's crocodile, which causes typhoid fever (Colomba et al. 2008). Listeria monocytogenes, Yersinia enterocolitica, Shigella spp. and Escherichia coli found in the present study are also important food-borne pathogens that can cause enteric infections (Levine \& Vial 1988, Bottone 1997, Kotloff et al. 1999, Schlech 2000).

Furthermore, several of the bacteria found in the cloacal cavity of crocodiles in the present study are potential pathogens and could affect water quality and human health. Johnston et al. (2010) studied bacteria from the cloaca of the American alligator Alligator mississippiensis as well as bacteria from surface water samples from their habitat, and they found similar flora present in the cloaca of alligator and the water they inhabit. Johnston et al. (2010) concluded that alligators are a potential source of bacterial contamination of their aquatic habitat through the excretion of feces. Other studies have reported the presence of similar bacterial flora in crocodilians and the water they inhabit (Shotts et al. 1972, White et al. 1973, Flandry et al. 1989, Madsen 1994). In captivity, tank water was also contaminated with bacteria from crocodilian feces (Kennedy 1973, Flandry et al. 1989, Madsen 1994, Huchzermeyer 2003). Thus, bacteria found in the present study in American and Morelet's crocodiles can also be expected to be present in their respective environments, and several of these bacteria are opportunistic pathogens that may cause wound or enteric infections in humans during activities in water or mud (Pitlik et al. 1987, Keene et al. 1994, Vally et al. 2004, Noonburg 2005).

Therefore, the bacteria found in the oral and cloacal cavities in Morelet's and American crocodiles in this study are potential pathogenic agents for crocodiles, humans (and other victims of crocodile attacks such as domestic animals) and their environments. Presently, humans rely on a large array of antibiotics to fight bacterial infections, and several studies on crocodile attacks have presented a broad spectrum of prophylactic antibiotics that could be used for the treatment of crocodile bite wounds (Caldicott et al. 2005, Hertner 2006, Gruen 2009, Wamisho et al. 2009, Langley 2010). However, even though many bacteria are sensitive to antibiotics, some strains/species are unaffected by, or show resistance to, some antibiotics (Montgomery et al. 2002, Bergstrom \& Feldgarden 2008). Moreover, bacteria are rapidly evolving organisms that can become resistant to antibiotics within several years after widespread use 
of the antibiotic (Bergstrom \& Feldgarden 2008). Thus, future studies should involve experiments on sensitivity and resistance of bacteria found in crocodiles in Quintana Roo in order to improve the treatment of wounds in victims of crocodile attacks. This would also provide important information to use in the treatment of wound infections in crocodiles and domestic animals that develop from crocodile bites.

\section{CONCLUSION}

This study provides important information about bacterial flora carried by American and Morelet's crocodiles in Quintana Roo State, Mexico. These bacteria can be a potential threat for crocodile health during conditions of stress but also represent a threat to human health through crocodile attacks and bites, consumption of crocodile meat or by simply carrying out activities in crocodile habitat. The risk of bacterial contamination from crocodiles to humans could increase in the future owing to several factors, principally the accelerated destruction and fragmentation of habitat, which could lead to an augmentation of human-crocodile interactions. Information on bacteria diversity reported in this study could help in the choice of antibacterial products for cases of infection from bacteria of crocodile origin.

Acknowledgements. We thank J. Nevarez for his helpful comments on earlier versions of the manuscript and for English revision. We also thank J. G. Ricalde for providing information on bacteria species. The map was produced by $\mathrm{H}$. Weissenberger. We also thank H. González-Cortés and the Fundación de Parques y Museos de Cozumel for providing support for field work. We are also very grateful to Banco Chinchorro Biosphere Reserve (CONANP) and its staff for their assistance and support during field work. This study was funded by El Colegio de la Frontera Sur (ECOSUR), Dirección General de Educación Superior Tecnológica (Project 2581.09-P) and Africam Safari. P.C. was awarded with a fellowship from the postdoctoral fellowship program of the Universidad Nacional Autónoma de Mexico. Scientific research permits were issued by the Secretaría de Medio Ambiente y Recursos Naturales (SEMARNAT) of Mexico (Oficio NÚM.SGPA/DGVS/02932/06 and Oficio NÚM. SGPA/DGVS/00724/10).

\section{LITERATURE CITED}

Anderson JR (1999) Characterization of the oral and cloacal flora of wild and farmed crocodiles (Crocodylus porosus and C. johnstoni) in Northern Australia. BSc (Hons) thesis, James Cook University, Townsville

Bergstrom CT, Feldgarden M (2008) The ecology and evolution of antibiotic-resistant bacteria. In: Stearns S, Koella J (eds) Evolution in health and disease, 2nd edn. Oxford University Press, Oxford, p 125-137
Bishop EJ, Shilton C, Benedict S, Kong F and others (2007) Necrotizing fasciitis in captive juvenile Crocodylus porosus caused by Streptococcus agalactiae: an outbreak and review of the animal and human literature. Epidemiol Infect 135:1248-1255

Blaylock RS (1999) Antibiotic use and infection in snakebite victims. S Afr Med J 89:874-876

> Bottone EJ (1997) Yersinia enterocolitica: the charisma continues. Clin Microbiol Rev 10:257-276

Brazaitis PE (1968) The determination of sex in living crocodilians. Br J Herpetol 4:54-58

$>$ Brook I (2009) Management of human and animal bite wound infection: an overview. Curr Infect Dis Rep 11: 389-395

Caldicott DGE, Croser D, Manolis C, Webb G, Britton A (2005) Crocodile attack in Australia: an analysis of its incidence and review of the pathology and management of crocodilian attacks in general. Wilderness Environ Med 16:143-159

Cantera K (2000) Student survives reptile attack in Mexico. Crocodile Spec Group Newsl 19:20

Cedeño-Vázquez JR, Ross JP, Calmé S (2006) Population status and distribution of Crocodylus acutus and $C$. moreletii in southeastern Quintana Roo, Mexico. Herpetol Nat Hist 10:53-66

Charruau P, Cedeño-Vázquez JR, Calmé S (2005) Status and conservation of the American crocodile (Crocodylus acutus) in Banco Chinchorro Biosphere Reserve, Quintana Roo, Mexico. Herpetol Rev 36:390-395

Charruau P, Thorbjarnarson JB, Hénaut Y (2010) Tropical cyclones and reproductive ecology of Crocodylus acutus Cuvier, 1807 (Reptilia: Crocodilia: Crocodylidae) on a Carribbean atoll in Mexico. J Nat Hist 44:741-761

Colomba C, Saporito L, Titone L (2008) Typhoid fever. In: Heggenhougen HK, Quah S (eds) International encyclopedia of public health, Vol 6. Academic Press, San Diego, CA, p 414-420

Cowan ST, Steel KL (1974) Manual for the identification of medical bacteria, 2nd edn. Cambridge University Press, Cambridge

Cupul-Magaña FG, Rubio-Delgado A, Reyes-Juárez A (2005) La mordida del cocodrilo americano (Crocodylus acutus), ¿es potencialmente séptica? Rev Biomed 16: 65-67

> Cupul-Magaña FG, Rubio-Delgado A, Reyes-Nuñez C, Torres-Campos E, Solís-Pecero LA (2010) Ataques de cocodrilo de río (Crocodylus acutus) en Puerto Vallarta, Jalisco, México: presentación de cinco casos. Cuad Med Forense 16:153-160

> Doering EJ III, Fitts CT, Rambo WM, Bradham GB (1971) Alligator bite. J Am Med Assoc 218:255-256

Erickson GM, Lappin AK, Vliet KA (2003) The ontogeny of bite-force performance in American alligator (Alligator mississippiensis). J Zool 260:317-327

Finger AG, Rainwater TR, McMurry ST, Platt SG, Mazzotti FJ (2002) Human-crocodile conflict in Belize: a summary. In: Crocodiles. Proceedings of the 16th working meeting of the crocodile specialist group. International Union for the Conservation of Nature, Gland, p 198-199

> Flandry F, Lisecki EJ, Domingue GJ, Nichols RL, Greer DL, Haddad RJ Jr (1989) Initial antibiotic therapy for alligator bites: characterization of the oral flora of Alligator mississippiensis. South Med J 82:262-266

Garcia ME, Lanzarot P, Costas E, Lopez Rodas V, Marín M, Blanco JL (2008) Isolation of Serratia fonticola from skin 
lesions in a Nile crocodile (Crocodylus niloticus) with an associated septicemia. Vet J 176:254-256

Garel A, Rainwater TR, Platt SG (2005) Triathlon champion attacked by crocodile in Belize. Crocodile Spec Group Newsl 24:8-10

Goldstein EJC, Citron DM, Merriam CV, Warren YA, Tyrell KL, Fernandez H (2002) In vitro activities of the desfluoro(6) quinolone BMS-284756 against aerobic and anaerobic pathogens isolated from skin and soft tissue animal and human bite wound infections. Antimicrob Agents Chemother 46:866-870

González-Cortés H (2007) Estudio poblacional de Crocodylus acutus (Cuvier, 1807) en el Refugio Estatal de Flora y Fauna Laguna de Colombia, Cozumel, Quintana Roo, México. Tesis de Licenciatura, Universidad Veracruzana, Córdoba

Gorden RW, Hazen TC, Esch GW, Fliermans CB (1979) Isolation of Aeromonas hydrophila from the American alligator, Alligator mississippiensis. J Wildl Dis 15:239-243

Gruen RL (2009) Crocodile attacks in Australia: challenges for injury prevention and trauma care. World J Surg 33: 1554-1561

Hernández-Hurtado H, Cupul-Magaña FG (1996) Presencia de Pseudomonas aeruginosa en cocodrilo de río: reporte de caso. Rev Biomed 7:245-248

> Hertner G (2006) Caiman bite. Wilderness Environ Med 17: 267-270

> Hoffmann FL, Romanelli PF (1998) Análise microbiológica da carne de jacaré do Pantanal (Caiman crocodilus yacare). Ciênc Tecnol Aliment 18:258-264

Huchzermeyer FW (2003) Crocodiles: biology, husbandry and diseases. CABI Publishing, Wallingford

> Jofré Morales L, Perret CP, Abarca KV, Solari VG, Olivares RC, Del P, López J (2006) Recomendaciones para el manejo de mordeduras ocasionadas por animales. Rev Chil Infectol 23:20-34

> Johnston MA, Porter DE, Rhodes GI, Webster LF (2010) Isolation of faecal coliform bacteria from the American alligator (Alligator mississippiensis). J Appl Microbiol 108: 965-973

Kämpfer P, Rauhoff O, Dott W (1991) Glycosidase profiles of members of the family Enterobacteriaceae. J Clin Microbiol 29:2877-2879

Keene WE, McAnulty JM, Hoesly FC, Williams LP Jr and others (1994) A swimming-associated outbreak of hemorrhagic colitis caused by Escherichia coli O157:H7 and Shigella sonnei. N Engl J Med 331:579-584

Kennedy ME (1973) Salmonella isolations from snakes and other reptiles. Can J Comp Med 37:325-326

Killian M, Bulow P (1976) Rapid diagnosis of Enterobacteriaceae. I. detection of bacterial glycosidases. Acta Pathol Microbiol Scand B Microbiol Immunol 84:245-251

Kotloff KL, Winickoff JP, Ivanoff B, Clemens JD and others (1999) Global burden of Shigella infections: implications for vaccine development and implementation of control strategies. Bull World Health Organ 77:651-666

- Kunimoto D, Rennie R, Citron DM, Goldstein EJC (2004) Bacteriology of a bear bite wound to a human: case report. J Clin Microbiol 42:3374-3376

Lamarque F, Anderson J, Fergusson R, Lagrange M, OseiOwusu I, Bakker L (2009) Human-wildlife conflict in Africa: causes, consequences and management strategies. FAO For Pap 157, FAO, Rome

Langley RL (2010) Adverse encounters with alligators in the United States: an update. Wilderness Environ Med 21: 156-163
Lazcano-Barrero MA (1996) Crocodile attacks in Cancun. Crocodile Spec Group Newsl 15:25-27

Levine MM, Vial P (1988) Escherichia coli that cause diarrhea. Indian J Pediatr 55:183-190

> Lovely CJ, Leslie AJ (2008) Normal intestinal flora of wild Nile crocodiles (Crocodylus niloticus) in the Okavango Delta, Botswana. J S Afr Vet Assoc 79:67-70

MacFaddin JF (2000) Biochemical tests for identification of medical bacteria, 3rd edn. Lippincott, Williams \& Wilkins, Baltimore, MD

Machkour-M'Rabet S, Hénaut Y, Charruau P, Gevrey M, Winterton P, Legal L (2009) Between introgression events and fragmentation, islands are the last refuge for the American crocodile in Caribbean Mexico. Mar Biol 156:1321-1333

Madsen M (1993) Microbial flora of frozen tail meat from captive Nile crocodiles (Crocodylus niloticus). Int J Food Microbiol 18:71-76

Madsen M (1994) Enumeration of salmonellae in crocodile pond water by direct plate counts and by the MPN technique. Water Res 28:2035-2037

> Madsen M, Hangartner P, West K, Kelly P (1998) Recovery rates, serotypes, and antimicrobial susceptibility patterns of Salmonellae isolated from cloacal swabs of wild Nile crocodiles (Crocodylus niloticus) in Zimbabwe. J Zoo Wildl Med 29:31-34

Magnino S, Colin P, Dei-Cas E, Madsen M and others (2009) Biological risks associated with consumption of reptile products. Int J Food Microbiol 134:163-175

Marlin JA, Marlin KK, Platt SG (1995) A documented case of an attack by Morelet's crocodile (Crocodylus moreletii) on man. Bull Chicago Herpetol Soc 30:165-167

Matushima ER, Ramos MCC (1995) Algunas patologias na criaçâo de jacarés no Brasil. In: Larriera A, Verdade LM (eds) La conservación y el manejo de caimanes y cocodrilos de América Latina, Vol 1. Fundación Banco Bico, Santo Tomé, Santa Fé, p 171-187

Mehrotra PK, Mathur BBL, Bhargava S, Choudhary S (2000) Mortality in gharial (Gavialis gangeticus) hatchlings at Jaipur zoo. Zoo's Print J 15:267-268

Mekisic AP, Wardill JR (1992) Crocodile attacks in the Northern Territory of Australia. Med J Aust 157: 751-754

Merchant M, Britton A (2006) Characterization of serum complement activity of saltwater (Crocodylus porosus) and freshwater (Crocodylus johnstoni) crocodiles. Comp Biochem Physiol A Mol Integr Physiol 143:488-493

> Merchant ME, Leger N, Jerkins E, Mills K, Pallansch MB, Paulman RL, Ptak RG (2006) Broad spectrum antimicrobial activity of leukocyte extracts from the American alligator (Alligator mississippiensis). Vet Immunol Immunopathol 110:221-228

> Mermin J, Hutwagner L, Vugia D, Shallow S and others (2004) Reptiles, amphibians, and human Salmonella infection: a population-based, case-control study. Clin Infect Dis 38:S253-S261

Montgomery JM, Gillespie D, Sastrawan P, Fredeking TM, Stewart GL (2002) Aerobic salivary bacteria in wild and captive komodo dragons. J Wildl Dis 38:545-551

> Murphey DK, Septimus EJ, Waagner DC (1992) Catfishrelated injury and infection: report of two cases and review of the literature. Clin Infect Dis 14:689-693

Murray PR, Baron EJ, Pfaller MA, Tenover FC, Yolken RH (1999) Manual of clinical microbiology, 7th edn. American Society for Microbiology, Washington, DC 
Narayana S, Metz LI, Kowalski TJ (2008) African tick bite fever and crocodile meat-associated salmonellosis coinfection in a returning traveler. Gunderson Lutheran Med J 5:17-18

Noonburg GE (2005) Management of extremity trauma and related infections occurring in the aquatic environment. J Am Acad Orthop Surg 13:243-253

Novak SS, Seigel RA (1986) Gram-negative septicemia in American alligators (Alligator mississippiensis). J Wildl Dis 22:484-487

Peucker SKJ, Davis BM, van Barneveld RJ (2005) Crocodile farming research: hatching to harvest. Rural Industries Research and Development Corporation, Barton

Pitlik S, Berger SA, Huminer D (1987) Nonenteric infections acquired through contact with water. Rev Infect Dis 9: 54-63

Pooley AC, Hines T, Shields J (1989) Attacks on humans. In: Ross CA, Garnett S (eds) Crocodiles and alligators. Facts on file, New York, NY, p 172-187

Quinn PJ, Carter ME, Markey BK, Carter GR (eds) (1994) Clinical veterinary microbiology. Wolfe-Mosby Publishing, London

Raynor AC, Bingham HG, Caffee HH, Dell P (1983) Alligator bites and related infections. J Fla Med Assoc 70:107-110

Revol B (1995) Crocodile farming and conservation, the example of Zimbabwe. Biodivers Conserv 4:299-305

Rickard MW, Thomas AD, Bradley S, Forbes-Faulkner J, Mayer RJ (1995) Microbiological evaluation of dressing procedures for crocodile carcasses in Queensland. Aust Vet J 72:172-176

Schlech WF III (2000) Foodborne listeriosis. Clin Infect Dis 31:770-775

Shotts EB Jr, Gaines JL Jr, Martin L, Prestwood AK (1972) Aeromonas-induced deaths among fish and reptiles in an eutrophic inland lake. J Am Vet Med Assoc 161:603-607

Schumacher J, Cardeilhac PT (1990) Mycotic infections of egg membranes in the American alligator (Alligator mis-

Editorial responsibility: Alex Hyatt,

Geelong, Victoria, Australia sissippiensis). Proc Int Assoc Aquat Anim Med 21: 138-140

Scott R, Scott H (1994) Crocodile bites and traditional beliefs in Korogwe district, Tanzania. Br Med J 309:1691-1692

Silva JSA, Mota RA, Pinheiro JWJ, Almeida MCS, Silva DR, Ferreira DRA, Azevedo JCN (2009) Aerobic bacterial microflora of broad-snouted caiman (Caiman latirostris) oral cavity and cloaca, originating from Parque Zoológico Arruda Câmara, Paraíba, Brazil. Braz J Microbiol 40: 194-198

Suárez MC, Pérez L, Murcia LM, Sarmiento LM, Casilimas S (2000) Intoxicación alimentaria por consumo de carne de caimán negro (Melanosuchus niger) en el internado indígena de Nazareth, Amazonas, abril de 1997. Biomédica 20:42-48

Thomas AD, Forbes-Faulkner JC, Speare R, Murray C (2001) Salmonelliasis in wildlife from Queensland. J Wildl Dis 37:229-238

Vally H, Whittle A, Cameron S, Dowse GK, Watson T (2004) Outbreak of Aeromonas hydrophila wound infections associated with mud football. Clin Infect Dis 38: 1084-1089

Vanwersch K (1998) Crocodile bite injuries in Southern Malawi. Trop Doct 28:221-222

Vyas R (2010) Mugger (Crocodylus palustris) population in and around Vadodara city, Gujarat state, India. Russ J Herpetol 17:43-50

> Wamisho BL, Bates J, Tompkins M, Islam R, Nyamulani N, Ngulube C, Mkandawire NC (2009) Ward round-crocodile bites in Malawi: microbiology and surgical management. Malawi Med J 21:29-31

> White FH, Simpson CF, Williams LE (1973) Isolation of Edwardsiella tarda from aquatic animal species and surface waters in Florida. J Wildl Dis 9:204-208

Ziegler T, Olbort S (2007) Genital structures and sex identification in crocodiles. Crocodile Spec Group Newsl 26: 16-17

Submitted: May 19, 2011; Accepted: October 17, 2011 Proofs received from author(s): January 31, 2012 